selectivity. The restriction of selectivity is mainly compelled by the specific characteristics of large-scale multi-agent systems. In such settings, it is reasonable to assume that the overhearing resources will be essentially limited, thus allowing the overhearing agent to overhear only a subset of inter-agent communications. Most previous investigations on overhearing ignore the limitation of selectivity, assuming that all relevant interagent communications can be overheard. In contrast, our work provides an empirical study of selective overhearing committed by both centralized and distributed teams of collaborative overhearing agents.

\title{
Cognitively Inspired Decision Making for Software Agents: Integrated Mechanisms for Action Selection, Expectation, Automatization and Non-Routine Problem Solving
}

Candidate: Aregahegn Seifu Negatu

Institution: Computer Science Department, The University of Memphis, TN, USA

Supervisor: Stan Franklin

Year awarded: 2006

URL: http: / / ccrg.cs . memphis . edu /

doi:10.1017/S0269888909990245

\section{Abstract}

Despite impressive advances in the past decades, autonomous agents living in dynamic and unpredictable environments are typically equipped with simple decisionmaking mechanisms in their sense-decide-act routines. These agents deal mostly with one goal at a time. Thi research aspires to model, design and/or implement a sophisticated decision-making mechanism that selects the agent's next action with different levels of awareness: automatized skills, consciously mediated routine solutions and consciously deliberated non-routine solutions. Such a decision-making mechanism is presented in a 'conscious' software agent framework called IDA that implements Baars' Global Workspace Theory of consciousness. IDA integrates many computational and conceptual mechanisms, among which this research deals with its action selection, expectation, automatization and non-routine problem-solving modules.

The overarching continual task of an agent's intelligence is for the service of choosing, at each moment in time, the appropriate action in response to exogenous and endogenous stimuli. IDA's action selection mechanism (ASM) can interleave and prioritize actions of different and competing goal hierarchies. The ASM system is implemented as a domain-independent and reusable framework for behavior networks and is tested as a controller to a khepera robot operating in a real-world domain.

We humans have the amazing ability to learn a procedural task (e.g. walking) so well that we do not need to think about the task consciously in order to accomplish it. This ability is what we call automatization. Once a task has been automatized, there is no need for attention to be paid to its execution unless the expected result does not occur. At failure of expectation, deautomatization process temporarily disables the automatization effects and 'conscious' control plays a role to deal with the failure situation. We implement the automatization and deautomatization cognitive functions as a self-organizing system in the IDA framework.

Non-routine problem solving is the ability to devise unexpected, and often clever, solutions to problems that have never been encountered before. We will present a detailed design and specification of a non-routine problem-solving mechanism as a special goal context hierarchy that guides a deliberative solution search process, which we will discuss in IDA's cognitive cycle.

\footnotetext{
Autonomy vs. Conformity: An Institutional Perspective on Norms and Protocols

Candidate: Huib Aldewereld

Institution: Intelligent Systems Group, Institute of Computer and Information Sciences, Universiteit Utrecht, The Netherlands

Supervisors: John-Jules Meyer and Frank Dignum

Year awarded: 2007

URL: http: / / igitur-archive.1ibrary.uu.nl/dissertations /2007-0604-200758/

doi:10.1017/S0269888909990257
}

\begin{abstract}
The research presented in this thesis is part of the ToKeN project ANITA (Administrative Normative Information Transaction Agents). In this domain, the main challenges concern both the shortage of information (not being able to find legally relevant data that should be available) as well as the abundance of information (e.g., violating privacy rights). Although the agents decide autonomously whether to share information based on (local) norms, a global frame was needed for the enforcement of (global) norms given by laws and regulations of the domain. In most software and agent methodologies, such regulations are seen only as extra requirements in the analysis phase, and are thus hard-coded into the software or agents themselves. If, however the regulations change, all design steps have to be checked and all code verified to ensure
\end{abstract}

compliance to the new regulations. The alternative is to have an explicit representation of the norms, for example, by the introduction of an electronic institution, but this approach requires some form of enforcement to ensure the compliance instead. The introduction of an electronic institution in highly regulated domains such as the ANITA scenario requires us to solve issues related to the abstractness of human regulations, the lack of operational information and the implementation of norm enforcement from an institutional perspective. In this thesis, we solve these problems by the introduction of a framework for making the connections between the norms and the practice explicit. To ensure that none of the norms derived from law are violated, an enforcement mechanism is presented based on monitoring and punishing rather than 